\title{
A supersingular coincidence
}

\section{G. K. Sankaran ${ }^{1}(\mathbb{1}$}

Received: 11 July 2021 / Accepted: 15 October 2021 / Published online: 20 December 2021

(c) The Author(s) 2021

\section{Abstract}

The 15 primes $2,3,5,7,11,13,17,19,23,29,31,41,47,59,71$ are called the supersingular primes: they occur in several contexts in number theory and also, strikingly, they are the primes that divide the order of the Monster. It is also known that the moduli space of $(1, p)$-polarised abelian surfaces is of general type for these primes. In this note, we explain that apparently coincidental fact by relating it to other numbertheoretic occurences of the supersingular primes.

Keywords Supersingular primes $\cdot$ Abelian surface $\cdot$ Jacobi form $\cdot$ Monster

Mathematics Subject Classification 11F50 11F46 $14 \mathrm{~K} 10$

\section{Introduction}

The list of 15 primes

$$
\mathscr{S}=\{2,3,5,7,11,13,17,19,23,29,31,41,47,59,71\}
$$

known as the supersingular primes (https://oeis.org/A002267) appears in several different contexts. Here are five of them.

1. $p \in \mathscr{S}$ if and only if $p$ divides the order of the Monster sporadic simple group.

2. $p \in \mathscr{S}$ if and only if $g\left(X_{0}(p)^{+}\right)=0$, where $X_{0}(p)^{+}=\mathbb{H} / \Gamma_{0}(p)^{+}$is the modular curve associated with the group $\Gamma_{0}(p)^{+}<\mathrm{GL}(2, \mathbb{Q})$.

3. $p \in \mathscr{S}$ if and only if the supersingular values of the $j$-invariant all lie in $\mathbb{F}_{p}$.

4. $p \in \mathscr{S}$ if and only if the space $J_{2, p}^{\text {cusp }}$ of Jacobi cusp forms of weight 2 and index $p$ is of dimension 0 .

5. If $p \notin \mathscr{S}$ then the moduli space $\mathscr{A}_{p}$ of complex abelian surfaces with a polarisation of type $(1, p)$ is of general type.

G. K. Sankaran

G.K.Sankaran@bath.ac.uk

1 Department of Mathematical Sciences, University of Bath, Bath BA2 7AY, UK 
Of these, (1)-(3) are described in [13], where the equivalence of the conditions in (2) and (3) is proved. In [13] a prize (a bottle of Jack Daniels) is offered for an explanation of why the condition in (1) is equivalent to those in (2) and (3): it is still unclaimed. Monstrous moonshine [2] provides a route from (1) to (2), but not in the other direction: see [3] for further details and [1] for some recent developments.

This note is primarily about (5). The proof that $\mathscr{A}_{p}$ is of general type for $p \notin \mathscr{S}$ is due to Erdenberger [5], and specialists in moduli of abelian surfaces are occasionally asked to explain the apparent coincidence [11]. In fact the answer consists of a series of well-known facts, but because they are not all well known to the same people, the question still recurs. The purpose of this note is to set the answer out clearly.

\section{Moduli of abelian surfaces}

An abelian surface equipped with a polarisation of type $(1, d)$ (for $d \in \mathbb{N}$ ) may be thought of as a complex torus $\mathbb{C}^{2} / \Lambda$, where $\Lambda \subset \mathbb{C}^{2}$ is the subgroup (lattice) generated by the columns of $\Omega=\left(I_{d}, \tau\right)$ for

$$
I_{d}:=\left(\begin{array}{ll}
1 & 0 \\
0 & d
\end{array}\right), \quad \tau=\left(\begin{array}{ll}
\tau_{1} & \tau_{2} \\
\tau_{2} & \tau_{3}
\end{array}\right) \in \mathbb{H}_{2}=\left\{\tau={ }^{t} \tau \in M_{2 \times 2}(\mathbb{C}) \mid \operatorname{Im} \tau>0\right\}
$$

The paramodular group

$$
\Gamma_{d}=\left\{\gamma \in \mathrm{GL}(4, \mathbb{Q}) \mid{ }^{t} \gamma\left(\begin{array}{cc}
0 & I_{d} \\
-I_{d} & 0
\end{array}\right) \gamma=\left(\begin{array}{cc}
0 & I_{d} \\
-I_{d} & 0
\end{array}\right)\right\}
$$

acts on the Siegel upper half-plane $\mathbb{H}_{2}$ by fractional linear transformations

$$
\left(\begin{array}{ll}
A & B \\
C & D
\end{array}\right): \tau \longrightarrow(A \tau+B)(C \tau+D)^{-1}
$$

This group action is properly discontinuous and the quotient $\mathscr{A}_{d}:=\mathbb{H}_{2} / \Gamma_{d}$ is a coarse moduli space for $(1, d)$-polarised abelian varieties. It is a quasi-projective variety, and one may ask for its Kodaira dimension, or more precisely, for the Kodaira dimension $\kappa\left(Y_{d}\right)$ of a desingularisation $Y_{d}$ of a projective compactification $\overline{\mathscr{A}}_{d}$. For more on this and related spaces, see [12].

In practice one expects that $\mathscr{A}_{d}$ is of general type, i.e. $\kappa\left(Y_{d}\right)=3$, except for some small values of $d$. Very loosely, this is because $k$-fold differential forms on $Y_{d}$ correspond to suitable modular forms of weight $3 k$ for $\Gamma_{d}$, and these become abundant as $d$ grows at least for $k$ sufficiently divisible. However, not every modular form of weight $3 k$ will do: obstructions come from the boundary $\overline{\mathscr{A}}_{d} \backslash \mathscr{A}_{d}$ and from the branching of $\mathbb{H}_{2} \rightarrow \mathscr{A}_{d}$.

The obstructions at the boundary may be overcome by using the low-weight cusp form trick [7]: if we can find a cusp form $f_{2}$ of weight 2 for $\Gamma_{d}$ then we may consider modular forms $f$ of weight $3 k$ of the form $f=f_{2}^{k} f_{k}$, where $f_{k}$ is a modular form 
of weight $k$. These are also abundant, if $d$ and $k$ are large enough, and because they vanish to high order at the boundary, the associated differential forms extend.

The branching behaviour has to be analysed separately, and it depends on the factorisation of $d$. For that reason much work in this direction has concentrated, for simplicity, on the case $d=p$ prime. The case $d=p^{2}$ has some simplifying features and was treated in $[8,14]$.

By this method, it was shown in [15] that $\mathscr{A}_{p}$ is of general type for $p>173$. Because of the inefficient compactification used there, the effective constraint on $p$ came from the branching, so all that was necessary was to verify that a weight 2 cusp form exists for all $p>173$. Such a form may be obtained by lifting a Jacobi cusp form of weight 2 and index $p$ according to Gritsenko ([7, Theorem 3], [6]). The dimension of the space of Jacobi cusp forms is computed in [4,17] and in this case it takes the form $[7,15]$

$$
\operatorname{dim} J_{2, p}^{\text {cusp }}=\sum_{j=1}^{p}\left\lfloor\frac{1+j}{6}\right\rfloor-\delta_{6}(j)-\left\lfloor\frac{j^{2}}{4 p}\right\rfloor
$$

where $\delta_{6}(j)=1$ if $6 \mid j$ and 0 otherwise. This is positive for all $p>173$.

Erdenberger [5] found a better compactification and was able to reduce the condition imposed by the branching from $p>173$ to $p \geq 37$, so that the existence of the Jacobi form becomes the effective constraint. Then, it is easy to compute from the formula above that $p \in \mathscr{S}$ exactly when no Jacobi cusp form of weight 2 and index $p$ exists, i.e. when the condition in (4) holds.

It is not necessarily to be expected that $\mathscr{A}_{p}$ is of general type exactly when $p \notin \mathscr{S}$. The method of proof of [5] fails for $p \in \mathscr{S}$, as we shall see, and it is known that $\mathscr{A}_{d}$ is unirational (so in particular not of general type) for some small values of $d$, including all primes $p \leq 11$ : see [9]. However, if $p \geq 13$, nothing currently excludes the possibility that $\mathscr{A}_{p}$ is of general type.

On the other hand, the possibility that $\mathscr{A}_{p}$ is unirational, other than in the known cases $p \leq 11$, is excluded. Gritsenko [6] showed that $\mathscr{A}_{d}$ has non-negative Kodaira dimension, so is not uniruled, for all $d \geq 13$, prime or not, except possibly for $d=14$, $15,16,18,20,24,30,36$. Of these, the cases $d=14,16,18,20$ have since been settled in [10] (such $\mathscr{A}_{d}$ are in fact unirational) and only for $d=15,24,30,36$ is nothing known about the Kodaira dimension of $\mathscr{A}_{d}$.

\section{Modular forms}

Since we have now established a connection between (4) and (5), to achieve a moderately satisfactory explanation of the apparently coincidental appearance of $\mathscr{S}$ in (5) we should show, without direct computation, that the conditions in (2) and (4) are equivalent. (A fully satisfactory explanation would also involve (1): this we are not able to give.) This is well known among specialists in Jacobi forms, and follows easily from a small part of [17]. 
It is shown in [17] that the space $J_{k, d}$ of Jacobi forms of weight $k$ and index $d$ is isomorphic (even as a Hecke module) to a certain subspace $\mathfrak{M}_{2 k-2}^{-}(d)$ of the space $M_{2 k-2}(d)$ of modular forms of weight $2 k-2$ for $\Gamma_{0}(d)$. This subspace is defined by $\mathfrak{M}_{2 k-2}^{-}(d)=M_{2 k-2}^{-}(d) \cap \mathfrak{M}_{2 k-2}(d)$, where $M_{2 k-2}^{-}(d)$ is the space of weight $2 k-2$ modular forms for $\Gamma_{0}(d)$ that satisfy an extra condition on the behaviour under the Fricke involution $w: \tau \mapsto \frac{-1}{d \tau}$, namely

$$
f\left(\frac{-1}{d \tau}\right)=(-1)^{k} d^{k-1} \tau^{2 k-2} f(\tau) .
$$

In our case $(k=2$ and $d=p)$ this is equivalent to saying that $f$ is a modular form of weight 2 for the group $\Gamma_{0}(p)^{+}<\operatorname{GL}(2, \mathbb{Q})$ generated by $\Gamma_{0}(p)$ and $w=\left(\begin{array}{cc}0 & 1 \\ -d & 0\end{array}\right)$. See, for example, the definition of automorphic form in [16, Chapter 2]. So if there is a weight 2, index $p$ Jacobi form, then $\Gamma_{0}(p)^{+}$has a weight 2 modular form.

Conversely, inspecting the definition of $\mathfrak{M}$ in [17] we find that there are no other conditions for $p$ prime: simply $\mathfrak{M}_{2 k-2}(p)=M_{2 k-2}(p)$. This can be seen at once, for instance, from [17, Eq. (4), p. 116], since $\mathfrak{M}_{2}(1) \subset M_{2}(1)=0$. In other words, the space of Jacobi forms in this case is isomorphic exactly to the space of weight 2 modular forms for $\Gamma_{0}(p)^{+}$. Moreover, the isomorphism respects cusp forms: see [17, Theorem 5].

We remark that for $k=2$ and $p$ square-free (in particular for $p$ prime) there are no Eisenstein series, so the condition (3) is equivalent to the same statement but with $J_{2, p}^{\text {cusp }}$ replaced by $J_{2, p}$.

However, Ogg [13] shows that the modular curve $X_{0}(p)^{+}$corresponding to $\Gamma_{0}(p)^{+}$ is of genus 0 precisely for $p \in \mathscr{S}$, i.e. he shows (2). One can compute the dimension of the space of weight 2 forms from the formulae given in [16, Theorem 2.23]: it is $g+m-1$, where $g$ is the genus of $X_{0}(p)^{+}$and $m$ is the number of cusps. Because $p$ is prime, the curve $X_{0}(p)$ has two cusps, which are interchanged by the Fricke involution; so $m=1$, and so the space of modular forms for $\Gamma_{0}(p)^{+}$has dimension $g$ (i.e. they are all cusp forms, as one should also expect from the remark above). So for $p \in \mathscr{S}$, there can be no weight 2 , index $p$ Jacobi forms; so we definitely cannot prove that $\mathscr{A}_{p}$ is of general type result by the methods of $[5,15]$ for any $p \in \mathscr{S}$.

Open Access This article is licensed under a Creative Commons Attribution 4.0 International License, which permits use, sharing, adaptation, distribution and reproduction in any medium or format, as long as you give appropriate credit to the original author(s) and the source, provide a link to the Creative Commons licence, and indicate if changes were made. The images or other third party material in this article are included in the article's Creative Commons licence, unless indicated otherwise in a credit line to the material. If material is not included in the article's Creative Commons licence and your intended use is not permitted by statutory regulation or exceeds the permitted use, you will need to obtain permission directly from the copyright holder. To view a copy of this licence, visit http://creativecommons.org/licenses/by/4.0/. 


\section{References}

1. Aricheta, V.M.: Supersingular elliptic curves and moonshine. SIGMA Symmetry Integr. Geom. Methods Appl. 15, 17 (2019)

2. Borcherds, R.E.: Monstrous moonshine and monstrous Lie superalgebras. Invent. Math. 109, 405-444 (1992)

3. Duncan, J.F.R., Ono, K.: The Jack Daniels problem. J. Number Theory 161, 230-239 (2016)

4. Eichler, M., Zagier, D.: The Theory of Jacobi Forms. Progress in Mathematics, vol. 55. Birkhäuser Boston, Inc., Boston (1985)

5. Erdenberger, C.: The Kodaira dimension of certain moduli spaces of Abelian surfaces. Math. Nachr. 274-275, 32-39 (2004)

6. Gritsenko, V.: Irrationality of the moduli spaces of polarized Abelian surfaces. Int. Math. Res. Not. 6, 235-243 (1994)

7. Gritsenko, V.: Irrationality of the moduli spaces of polarized abelian surfaces. Abelian varieties (Egloffstein, 1993), 63-84, de Gruyter, Berlin (1995)

8. Gritsenko, V., Sankaran, G.K.: Moduli of Abelian surfaces with a (1, $\left.p^{2}\right)$ polarisation. Izv. Ross. Akad. Nauk. Ser. Mat. 66, 19-26 (1997)

9. Gross, M., Popescu, S.: The moduli space of $(1,11)$-polarized Abelian surfaces is unirational. Compos. Math. 126, 1-23 (2001)

10. Gross, M., Popescu, S.: Calabi-Yau three-folds and moduli of abelian surfaces II. Trans. Am. Math. Soc. 363, 3573-3599 (2011)

11. He, Y.-H., McKay, J.: Sporadic and exceptional. arXiv:1505.06742, (2015)

12. Hulek, K., Kahn, C., Weintraub, S.: Moduli Spaces of Abelian Surfaces: Compactification, Degenerations, and Theta Functions. Expositions in Mathematics, vol. 12. de Gruyter, Berlin (1993)

13. Ogg, A.: Automorphismes de courbes modulaires. Séminaire Delange-Pisot Poitou (16e année: 1974/75), Théorie des nombres, Fasc. 1, Exp. No. 7, 8 pp. Secrétariat Mathématique, Paris (1975)

14. O'Grady, K.: On the Kodaira dimension of moduli spaces of Abelian surfaces. Compos. Math. 72, 121-163 (1989)

15. Sankaran, G.K.: Moduli of polarised Abelian surfaces. Math. Nachr. 188, 321-340 (1997)

16. Shimura, G.: Introduction to the arithmetic theory of automorphic functions. Kanô Memorial Lectures, No. 1. Publications of the Mathematical Society of Japan 11. Iwanami Shoten, Publishers, Tokyo; Princeton University Press, Princeton, N.J. (1971)

17. Skoruppa, N., Zagier, D.: Jacobi forms and a certain space of modular forms. Invent. Math. 94, 113-146 (1988)

Publisher's Note Springer Nature remains neutral with regard to jurisdictional claims in published maps and institutional affiliations. 